\title{
PRIMARY INTRAOSSEOUS MUCOEPIDERMOID CARCINOMA OF THE MAXILLA
}

\author{
S. M. NALLAMILLI ${ }^{1}$, R. TATAPUDI ${ }^{2}$, R. S. REDDY ${ }^{2}$, M. RAVIKANTH ${ }^{3}$ and N. RAJESH ${ }^{2}$ \\ ${ }^{1}$ Department of Oral Medicine and Radiology, Drs. Sudha and Nageswara Rao Siddhartha Institute of Denta \\ Sciences, Chinaoutapalli, Gannavaram Mandalam, Andhra Pradesh - $521286{ }^{2}$ Department of Oral medicine \\ and Radiology, Vishnu Dental College, Vishnupur Bhimavaram, Andhra Pradesh - 534202 ${ }^{3}$ Department of \\ Oral and Maxillofacial Pathology,Vishnu Dental College, Vishnupur Bhimavaram, Andhra Pradesh - 534202
}

DOI: $h t t p: / / d x . d o i . o r g / 10.4314 / g m j . v 49 i 2.10$

Corresponding author: Dr. Sai Madhavi Nallamilli

E-mail: shiny.dent@gmail.com

Conflict of interest: None declared

\section{SUMMARY}

Primary intraosseous mucoepidermoid carcinoma (PIOC) is an infrequent malignancy of the head and neck that exhibits diverse biological behavior. The rareness of the location for a salivary gland tumor and the clinical and radiographic manifestations, suggestive of an odontogenic lesion often pose a diagnostic challenge. We hereby report such a unique case of central mucoepidermoid carcinoma that presented in an ambiguous manner, developing in the maxilla and intruding into the adjacent vital structures, adding a literature review.

Keywords: Mucoepidermoid carcinoma, salivary gland malignancy, maxilla, CT imaging, histopathologic picture

\section{INTRODUCTION}

Mucoepidermoid carcinoma (MEC), comprising one third of all salivary malignancies, is considered the most common malignant salivary gland tumor. ${ }^{1}$ Central variant of MEC is quite rare but a well-known entity affecting the jaw bones. It has been reported in the first to seventh decade, and is slightly more common in women and the posterior mandible. ${ }^{2-6}$ We hereby report a vigorous case of high grade mucoepidermoid carcinoma, that presented with a large lesion in the maxilla extending to involve the maxillary sinus and nasal cavity.

\section{CASE REPORT}

A 36 year old male patient reported to the Department of Oral Medicine and Radiology with a chief complaint of swelling and pain in the right upper back teeth region. History revealed a spontaneous onset of the swelling 2 months back, which was initially small and gradually increased to present size with no associated symptoms.
He gave an additional history of occasional, dull pain in relation to a decayed tooth in the same region since 2 days, which subsided on taking pain killers. There was no relevant medical or personal history.

On extra-oral examination, a diffuse swelling involving the right middle third of the face extending superoinferiorly from the infraorbital margin to an imaginary line drawn laterally from the corner of the mouth, mediolaterally from the lateral border of the nose to an imaginary line drawn inferiorly from the outer canthus of the eye. The surface of the swelling was smooth, with color similar to adjacent mucosa. No secondary changes were evident. On palpation, there was no local rise in temperature, and the overlying skin was pinchable. The swelling was firm in consistency and non-tender. Regional submandibular lymph nodes were palpable bilaterally, single in number, firm in consistency, nontender and movable.

Intraorally, a well-defined swelling was noted involving the right maxilla and vestibule, approximately measuring $5 \mathrm{~cm}$ by $3 \mathrm{~cm}$ in size, extending medio-laterally from the mid-palatine raphae, obliterating the buccal vestibule and extending $0.5 \mathrm{~cm}$ inferiorly on to the buccal mucosa; anteroposteriorly from mesial margin of 13 to distal margin of 18. Surface of the swelling was irregular, with color similar to adjacent mucosa and demonstrated raised borders. On palpation, the consistency was firm, while appearing fluctuant at the palatal aspect of 13,14 and was non tender. Hard tissue examination revealed deep dental caries with respect to 15 , root stumps in relation to $18,28,38$, partial edentulousness in the region of 16 , 37,47 and a fair oral hygiene status. 
Based upon the history and clinical presentation, a provisional diagnosis of benign odontogenic tumour involving right maxilla was made. A list of reactive and neoplastic lesions was included in the differential diagnosis.

On radiographic examination, orthopantomograph revealed a radiolucency involving enamel, dentin and pulp in relation to 15 , an irregular osteolytic lesion in the right maxilla extending antero-posteriorly from 13 to 16 region, which superiorly caused erosion of the maxillary sinus floor and an increased radiodensity within the respective sinus, and extended inferiorly up to the alveolus in relation to $13,14,15,16$. Intraoral periapical radiographs and para nasal sinus view confirmed the same respectively. Computed tomography imaging revealed bone destruction with the epicenter in the right maxilla and erosion of the lateral wall of the maxillary sinus with an increased radiodensity in the respective sinus and the adjoining nasal cavity; likewise, the contra-lateral maxillary sinus and nasal cavity also demonstrated an increased radiodensity peripherally (Figure 1).

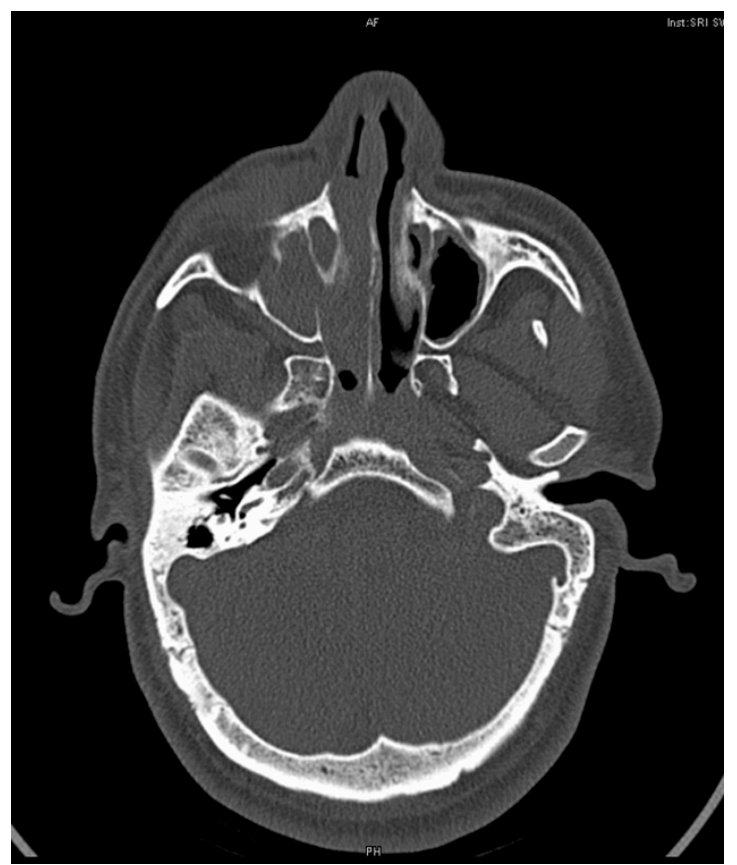

Figure 1 Axial computed tomography image revealing a destructive lesion in the left maxilla

Fine needle aspiration cytology yielded a negative aspiration. Incisional biopsy was performed from the buccal vestibule. Histopathological specimen revealed sheets of dysplastic glandular epithelial cells along with pseudocystic spaces. Solid nests of epidermoid cells with few intermediate cells are seen.
The epidermoid cells showed prominent nuclear pleomorphism and abnormal mitotic figures. Few areas exhibited squamous metaplasia of ductal epithelial cells and keratin pearl formation. Perineural invasion and necrosis is seen in few areas. Group of normal mucous acini are evident in one of those areas. The connective tissue stroma shows loosely arranged collagen fibres with areas of degeneration and haemorrhage. Minimal inflammatory cell infiltrate such as few lymphocytes are seen. Underlying muscle fibres showed degeneration (Figure 2).

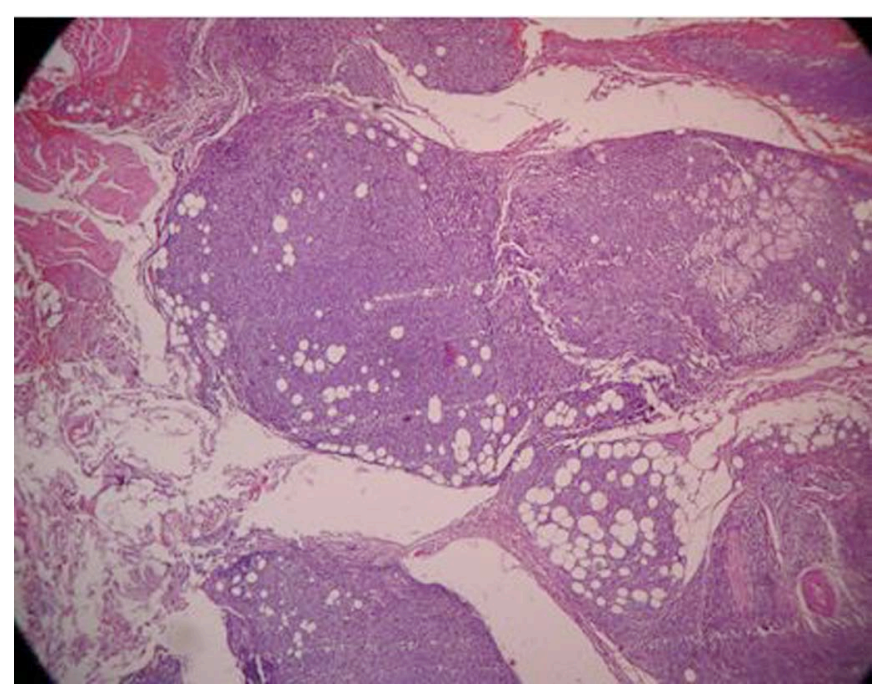

Figure 2 Histopathological specimen revealed sheets of dysplastic glandular epithelial cells along with pseudocystic spaces. Solid nests of epidermoid cells with few intermediate cells are seen.

PAS staining was positive in focal areas within the solid nests of epidermoid cells. Correlating all the evidence, a final diagnosis of high - grade Mucoepidermoid carcinoma involving right maxilla was arrived. Other investigations carried out as part of a metastatic work-up, showed no evidence of secondaries. The patient was subsequently referred to a higher regional oncology center for treatment.

\section{DISCUSSION}

Aberrant salivary gland neoplasms developing within jaws are exceptionally rare, accounting for $2 \%-4.3 \%$ of all MECs reported. Central MEC was first reported by Lepp in 1939 following which its pathogenesis, radiological and histopathological aspects have been extensively discussed. The chances of origin may be neoplastic transformation of the maxillary sinus lining or the entrapped minor salivary glands within the maxilla. 
In 2004, Kochaji and colleagues proposed a criteria for future case reports of the central MEC which were: ${ }^{1}$

(1) presence of an intact cortical plate

(2) radiological evidence of bone destruction

(3) histological confirmation

(4) positive mucin staining

(5) absence of primary lesion in the salivary gland

(6) exclusion of an odontogenic tumour

All the diagnostic criteria were fulfilled in the present case except for second which can be ascribed to chronicity of the lesion that allowed the lesion to grow to an extensive size. Brookstone and Huvos suggested a three-grade classification for intraosseous MEC. Grade 1, without expansion and rupture of cortical plate; grade 2, with expansion but without rupture of cortical plate; grade 3 , with rupture of cortical plates or presence of regional metastasis. ${ }^{1}$ The present case is pertinent to grade 3 .

Radiographic examination is vital to categorize a central jaw lesion and to determine the extent of the lesion with involvement or encroachment of adjacent vital structures. Panoramic radiography is simple and helpful for the lesions involving the jaw bones, however additional imaging with $\mathrm{CT}$ might be required in lesions involving the maxilla to discern the positive margins. In the present case, the involvement of the surrounding vital structures such as the maxillary sinus and nasal fossa will greatly dictate the treatment and prognosis.

Histopathological criteria for MEC diagnosis according to the criteria of Seifert and Sobin, and Auclair et al: (1) low grade: highly differentiated neoplasia with a predominance of macro- and microcysts. Presence of intermediate and mucin-producing cells (2) intermediate grade: predominance of intermediate cells and a few cysts. Presence of mucin-producing cells and islands of epidermoid cells (3) high grade: poorly differentiated neoplasia with predominance of intermediate and epidermoid cells in solid blocks. Mucin-producing cells are present. ${ }^{1}$ The findings in this case were allied to the high - grade variant of Muco-epidermoid carcinoma. ${ }^{7-11}$

Low grade mucoepidermoid carcinoma is treated surgically with local wide block, while the high grade tumours require wide block excision with radical neck dissection. Radiotherapy and chemotherapy are generally reserved for selected cases because of the long term adverse effects. ${ }^{12,13,14}$ Our patient was referred a regional oncology centre for treatment plan, but was sorrowfully unavailable for further follow-up.
Survival rate of patients with low-grade lesions is about $95 \%$ at 5 years while the rate drops to approximately $40 \%$ in case of high grade lesions. ${ }^{1}$

\section{CONCLUSION}

Central MEC in the maxilla is a rare entity whose features have been sparsely mentioned in the literature. It is frequently misdiagnosed, owing to the diverse clinical and radiographic presentations that are indicative of an odontogenic lesion.

The tumour is often treated inappropriately or late, eventually leading to recurrence. Advanced imaging greatly aids in timely diagnosis and to identify the involvement of adjacent vital structures, which dictates the management as well as the prognosis. Treated cases of central MEC should be monitored for a longer duration to identify late local recurrence or metastasis.

\section{REFERENCES}

1. Sumanth KN, Mainali A, Ongole R, Pai MR. Mucoepidermoid carcinoma: "A Mimicker"? Journal of Nepal Dental Association 2009;10:3134.

2. Raut DL, Khedkar SA. Primary intraosseous mucoepidermoid carcinoma of the maxilla: a case report and review of literature. Dentomaxillofacial Radiology 2009;38:163-168.

3. Zameer Phasha, Kapila Sharma, Sameer Choudhari, Sulabha AN. Mucoepidermoid carcinoma of palate mimicking vascular lesion: A case report. Journal of Dental Sciences and Research 2013;4:9 -12.

4. Fatih Sengul, Sera Simsek, Binali Cakur. Mucoepidermoid carcinoma in a minor salivary gland in a child. Case Reports in Dentistry Vol. 2013, Article ID 615948, doi.org/10.1155/2013/615948.

5. Seyedmajidi M, Foroughi R. Central Mucoepidermoid Carcinoma-A Case Report and Review of the Literature. J Clin Exp Oncol 2013;2:1-3.

6. Ines Velez, Maritzabel Hogge, Steven Kaltman, Eustorgio Lopez, Kurt Friedman. Central Mucoepidermoid Carcinoma: A Rare Entity Analysis of Five Cases. Journal of Research and Practice in Dentistry Vol. 2013, Article ID 530750, DOI: 10.5171/2013. 530750.

7. Ajay Bansal, Devi Charan Shetty, Harish Chandra Rai, Harkanwal Preet Singh. Primary intraosseous mucoepidermoid carcinoma of maxilla-a rare occurrence. e-Journal of Dentistry 2011;1:14-17.

8. Deepti Simon, Thara Somanathan, Ramdas K, Manoj Pandey. Central Mucoepidermoid carcinoma of mandible - A case report and review 
of the literature. World Journal of Surgical Oncology 2003;1:1-5.

9. Roland Giger, Paulette Mhawech, Francis Marchal, Willy Lehmann, Pavel Dulguerov. Mucoepidermoid carcinoma of stensen's duct: a case report and review of the literature. Head Neck 2005;27:829-833.

10. Kelgaonkar YU, Shaila Bangad, Sham Somani, Surabhi Chopra, Sachin Ingle. Mucoepidermoid carcinoma of parotid: a rare case report. International Journal of Basic and Applied Medical Sciences 2012; 2:136-8.

11. Nabil Kochaji, Annieta Goossens, Peter Bottenberg. Central Mucoepidermoid carcinoma: case report, literature review for missing and available information and guideline proposal for coming case reports. Oral Oncology Extra 2004;40:95-105.

12. Syed Asad Ali, Abdul Sattar Memon, Noshad Shaik, Abdul Ghani Soomro. Mucoepidermoid carcinoma of parotid presenting as unilocular cyst. J Ayub Med Coll Abbottabad 2008;20:141-42.

13. Horia Vulpe, Meredith Giuliani, David Goldstein, Bayardo Perez-Ordonez, Laura Dawson, Andrew Hope. Long term control of a maxillary sinus mucoepidermoid carcinoma with low dose radiation therapy: a case report. Radiation Oncology 2013;8:1-5.

14. John Williamson, Byron Simmons, Adel ElNaggar, Jeffrey Medeiros L. Mucoepidermoid Carcinoma Involving Warthin Tumor. A Report of Five Cases and Review of the Literature. $A m J$ Clin Pathol 2000;114:564-570. 